\title{
High expression of both desmoplastic stroma and epithelial to mesenchymal transi- tion markers associate with shorter survival in pancreatic ductal adenocarcinoma
}

\author{
Damián Sánchez-Ramírez, ${ }^{1,2}$ Rafael Medrano-Guzmán, ${ }^{3}$ Fernando Candanedo-González, ${ }^{4}$ \\ Jazmín De Anda-González, ${ }^{4}$ Luis Enrique García-Rios, ${ }^{3}$ Vadim Pérez-Koldenkova, ${ }^{5}$ \\ Marcos Gutiérrez-de la Barrera, ${ }^{6}$ Sara Rodríguez-Enríquez, ${ }^{7}$ Marco Velasco-Velázquez, ${ }^{8}$ \\ Silvia Cecilia Pacheco-Velázquez, ${ }^{7}$ Patricia Piña-Sánchez, ${ }^{9}$ Héctor Mayani, ${ }^{10}$ Alejandro Gómez-Delgado, ${ }^{11}$ \\ Alberto Monroy-García, ${ }^{12}$ Ana Karen Martínez-Lara, ${ }^{1}{ }^{2}$ uan José Montesinos ${ }^{1}$
}

${ }^{1}$ Mesenchymal Stem Cells Laboratory, Oncology Research Unit, Oncology Hospital, National Medical Center, IMSS, Mexico City; ${ }^{2}$ Programa de Doctorado de Ciencias Biomédicas, Universidad Nacional Autónoma de México (UNAM), Mexico City; ${ }^{3}$ Department of Sarcomas, Oncology Hospital, High Specialty Medical Unit (UMAE), National Medical Center, IMSS, Mexico City; ${ }^{4}$ Department of Pathology, Oncology Hospital, High Specialty Medical Unit (UMAE), National Medical Center, IMSS, Mexico City; ${ }^{5}$ National Laboratory of Advanced Microscopy-IMSS, National Medical Center, Siglo XXI IMSS, Mexico City; ${ }^{6}$ Oncology Research Unit, Oncology Hospital, National Medical Center, IMSS, Mexico City; ${ }^{7}$ Department of Biochemistry, National Institute of Cardiology Ignacio Chávez, Mexico City ${ }^{8}$ Department of Pharmacology and Peripheral Research Unit in Translational Biomedicine (CMN 20 de noviembre, ISSSTE), School of Medicine, UNAM, Mexico City; ${ }^{9}$ Molecular Oncology Laboratory, Oncology Research Unit, Oncology Hospital, National Medical Center, IMSS, Mexico City; ${ }^{10}$ Hematopoietic Stem Cells Laboratory, Oncology Research Unit, Oncology Hospital, National Medical Center, IMSS, Mexico City; ${ }^{11}$ Infectious and Parasitic Diseases, Medical Research Unit, Pediatric Hospital, National Medical Center, IMSS, Mexico City; ${ }^{12}$ Immunology and Cancer Laboratory, Oncology Research Unit, Oncology Hospital, National Medical Center (IMSS), Mexico City, Mexico

\begin{abstract}
Desmoplastic stroma (DS) and the epithelial-to-mesenchymal transition (EMT) play a key role in pancreatic ductal adenocarcinoma (PDAC) progression. To date, however, the combined expression of DS and EMT markers, and their association with variations in survival within each clinical stage and degree of tumor differentiation is unknown. The purpose of this study was to investigate the association between expression of DS and EMT markers and survival variability in patients diagnosed with PDAC. We examined the expression levels of DS markers alpha smooth muscle actin ( $\alpha$-SMA), fibronectin, and vimentin, and the EMT markers epithelial cell adhesion molecule (EPCAM), pan-cytokeratin, and vimentin, by immunohistochemistry using a tissue microarray in a retrospective cohort of 25 patients with PDAC. The results were examined for association with survival by clinical stage and by degree of tumor differentiation. High expression of DS markers $-\alpha$-SMA, fibronectin, and vimentin- was associated with decreased survival at intermediate and advanced clinical stages $(\mathrm{p}=0.006$ 0.03 ), as well as with both poorly and moderately differentiated tumor grades ( $\mathrm{p}=0.01-0.02)$. Interestingly, the same pattern was observed for EMT markers, i.e., EPCAM, pan-cytokeratin, and vimentin ( $\mathrm{p}=0.00008-0.03)$. High expression of DS and EMT markers within each clinical stage and degree of tumor differentiation was associated with lower PDAC survival. Evaluation of these markers may have a prognostic impact on survival time variation in patients with PDAC.
\end{abstract}

Key words: Pancreatic ductal adenocarcinoma; desmoplastic stroma; epithelial-to-mesenchymal transition; clinical stage; degree of tumor differentiation; survival.

Correspondence: Juan José Montesinos, PhD, Mesenchymal Stem Cells Laboratory, Oncology Research Unit, Oncology Hospital, National Medical Center, Guillermo Prieto 38, Depto C-102, Jamaica, Venustiano Carranza, 15800 Mexico City, Mexico. Tel: +52.5523156025. E-mail: montesinosster@gmail.com

Contributions: DSR, RMG, FCG, JAG, LEGR, VPK, MGB, SRE, MVV, SCPV, PPS, HM, AGD, AMG, AKML, conceptualized and designed the research project, carried out the practical work, formal analysis, and validation; DSR, JJM, performed the statistical analysis and writing - original draft preparation, review, editing and finalized the manuscript. All authors provided significant input in the manuscript, read and approved the final version of the manuscript and agreed to be accountable for all aspects of the work.

Funding: This work was funded by the Consejo Nacional de Ciencia y Tecnología (CONACyT) for support to J.J.M. (grant no. 272793) and IMSS support to J.J.M. (grant no. 1311 and 1731).

Conflict of interest: The authors declare that they have no competing interests, and all authors confirm accuracy.

Availability of data and materials: The datasets used and/or analyzed during the current study are available from the corresponding author on reasonable request.

Ethical Approval: The current study was approved by local Health Research Ethics Committee of the Oncology Hospital, National Medical Center, Century XXI, Mexican Institute of Social Security and recorded under protocol number R-20163602-37. All patients also provided informed consent for participating in this study. 


\section{Introduction}

Pancreatic cancer is one of the neoplasms with unfavorable prognosis, ranking first in lethality rate worldwide. ${ }^{1}$ Pancreatic ductal adenocarcinoma (PDAC) is the most frequent subtype, accounting for $85 \%$ of tumors of this organ. ${ }^{2,3}$ The American Joint Commission on Cancer (AJCC) tumor node metastasis (TNM) staging system is the standard diagnostic tool in which the disease process $^{4,5}$ and treatment guidelines ${ }^{6}$ are outlined. The AJCC has also incorporated the degree of tumor differentiation $(\mathrm{G})$ as a further criterion. ${ }^{7}$ Together with the TNM, the degree of tumor differentiation determines patient prognosis. ${ }^{6}$ The $\mathrm{G}$ criterion categorizes the tumor from well-differentiated (low grade) to undifferentiated (high grade); the more undifferentiated the tumor is, the more aggressive its biological behavior is. In PDAC, the histological grade is based on the extent of glandular differentiation. If more than $95 \%$ of a tumor is composed of glands, it is classified as well-differentiated; if 50-95\% of the tumor is composed of glands, it is moderately differentiated; and if less than $50 \%$ of it is composed of glands, it is poorly differentiated. ${ }^{6}$ Several studies have shown that the degree of tumor differentiation of pancreatic cancer is a key indicator of survival after tumor resection, ${ }^{7-10}$ although both AJCC criteria fail to explain the variability in the survival of patients with PDAC. Thus, $80 \%$ of patients with PDAC are diagnosed at advanced clinical stages and with a worse prognosis ${ }^{2,11}$ and the 5-year overall survival rate is 9\%.2,11,12 Furthermore, although the median survival for all clinical stages is 8 months, ${ }^{13}$ the reason why some patients survive up to 5 years, ${ }^{4}$ regardless of their clinical stages and degree of differentiation at diagnosis, remains unknown. . $^{40,13}$

PDAC is characterized as a prominent desmoplastic stroma (DS) defined by fibrotic tissue with an abundant extracellular matrix around the tumor tissue. ${ }^{14-16}$ The cells that promote the development of the DS are cancer-associated fibroblasts (CAFs). ${ }^{17}$ CAFs are activated during inflammation and carcinogenesis, acquiring a myofibroblast-like morphology and expressing proteins such as alpha smooth muscle actin ( $\alpha$-SMA), fibronectin, and vimentin, ${ }^{18,19}$ in addition to accumulating an excess of extracellular matrix molecules, cytokines, chemokines and growth factors. ${ }^{12,16-}$ 18,20-27 The DS helps to form more aggressive tumors, with an increased capacity for proliferation, invasion, and metastasis, as well as resistance to chemotherapy and radiotherapy. ${ }^{12,17,28,29}$ Several in vitro and in vivo studies have demonstrated that PDAC tumor cells stimulate the proliferation, migration, activation, and secretion of extracellular matrix proteins of CAFs. ${ }^{15,20,30}$ An example of this crosstalk between tumor cells and stromal cells is TGF$\beta$ which is secreted by tumor cells and considered one of the most important and well-studied factors inducing CAF activation. ${ }^{12}$ In turn, CAFs stimulate the proliferation, apoptosis, evasion, migration, invasion, and metastasis of PDAC tumor cells. ${ }^{14,20,23,31,32}$ As a result of these mutual cellular interactions, the process of epithelial-to-mesenchymal transition (EMT) of neoplastic cells is induced within the tumor microenvironment. ${ }^{19,21,22,29,33}$ During EMT, neoplastic cell epithelial markers such as the epithelial cell adhesion molecule (EPCAM) ${ }^{34}$ and cytokeratins ${ }^{33}$ are gradually lost, and mesenchymal markers such as vimentin ${ }^{33,35}$ are expressed. EMT is a reversible transdifferentiation process controlled by complex interactions between multiple signaling pathways such as TGF $\beta$, Wnt, and Notch, which converge to a network of specific transcription factors that convert cancer cells of epithelial differentiation into a more mesenchymal phenotypic state. ${ }^{36}$ The expression of mesenchymal markers favors the development of an invasive phenotype in which the tumor cell increases its migration, ${ }^{20}$ as shown in both in vitro ${ }^{21,37}$ and in vivo ${ }^{20,31}$ models. In fact, it has been shown that $\mathrm{T} \beta_{4}$ a peptide that regulate actin polymerization is pre- dominantly expressed at the invasion front in colorectal tumor cells undergoing EMT, which suggest a role for $\mathrm{T} \beta_{4}$ in invasion and metastasis. ${ }^{38}$ Therefore, microenvironment dynamics promotes tumor growth and invasion ${ }^{29,39}$ and EMT might be helpful as a biomarker in PDAC diagnosis and to be considered as a new therapeutic target in the management of this disease. ${ }^{40}$

The current prognostic stratification of PDAC based on the TNM and G criteria does not efficiently differentiate patients of different clinical stages who may survive for a period longer than the average time, ${ }^{41}$ including those at clinical stages III and IV. ${ }^{6,42}$ Consequently, new parameters must be included to help efficiently discriminate the survival of patients with PDAC within each clinical stage and tumor differentiation grade. Given that PDAC is characterized by an abundant DS and by the presence of EMT, biomarkers of both biological characteristics may be associated with PDAC aggressiveness and poor prognosis of patients with PDAC. Previous studies have associated the increased expression of DS markers, such as $\alpha-\mathrm{SMA}^{43,44}$ or EMT markers such as EPCAM, cytokeratins, and vimentin, ${ }^{45-49}$ with a tendency for low survival among patients with PDAC. However, the combined expression of markers of both characteristics and their association with variations in survival within each clinical stage and degree of tumor differentiation remain unknown. In this context, we hypothesize that quantitative evaluation of the expression levels of DS and EMT markers may improve the current stratification and provide a highly accurate prognosis. Therefore, in this pilot study, we evaluated the expression of markers of both characteristics and analyzed their association with survival variability in patients diagnosed with PDAC.

\section{Materials and Methods}

\section{Patients and samples}

All patients in this study were diagnosed with PDAC and underwent pancreatic resection and biopsy at the Oncology Hospital, National Medical Center, Century XXI (Centro Médico Nacional Siglo XXI [CMN Siglo XXI]) and Mexican Institute of Social Security (Instituto Mexicano del Seguro Social [IMSS]), Mexico City. Since PDAC is not as frequent as other tumors in our hospital such as in other International Medical Centers, ${ }^{1,50}$ we decide to study these markers in a first approach, as a pilot study. It has been suggested that the minimal number of samples must be calculated according to data from Cochran's sample size formula ${ }^{51}$ based on i) a level of $\alpha=0.001$; ii) a low margin of error $=3 \%$; and iii) a low disease frequency $=0.0014$. Thus, in our study 25 samples were collected and analyzed from 2007 to 2018. Our work is not the unique in the usage of reduced number of samples, in fact small cohorts of 7 and 10 patients have already been documented in other studies, respectively. ${ }^{52,53}$ Clinicopathological data were collected and included gender, age, stage $T$, stage $N$, stage $M$, TNM stages, degree of differentiation, overall survival, evolution time, the follow-up time of the first and the last medical consultation, the follow-up time of the disease onset at the last visit, desmoplasia, and type or surgery. Samples were fixed in formaldehyde and subsequently embedded in paraffin to prepare tissue microarrays (TMA).

The use of human samples was approved by the local Health Research Ethics Committee of the CMN Siglo XXI and IMSS and recorded under protocol number R-2016-3602-37.

\section{Tissue microarrays}

To minimize experimental variability and to ensure reproducible staining, TMA technology was applied using paraffin- 
embedded tissues. ${ }^{54}$ To corroborate the diagnosis of the pancreatic tumor samples, the hematoxylin and eosin stainings were re-evaluated by two expert pathologists, according to the 2010 World Health Organization classification. For the tumor samples, three replicates with a diameter of $1 \mathrm{~mm}$ were selected; these replicates were marked by our pathologist who selected areas with tumor tissue and desmoplastic stromal tissue for re-embedding into a recipient paraffin block using a tissue microarrayer (Chemicon International, Temecula, CA, USA). Once the TMAs were prepared, they were cut into serial $3-\mu \mathrm{m}$ sections. The microarray tissue slide contained approximately 75 cores corresponding to tumor samples from 25 patients, with three replicates each.

\section{Immunohistochemistry}

TMAs were stained with goat recombinant polyclonal anti- $\alpha-$ SMA antibody (1:250 dilution; Abcam, Cambridge, UK), rabbit recombinant polyclonal anti-fibronectin (1:1,000 dilution; Abcam), mouse recombinant monoclonal anti-vimentin $(1: 1,000$ dilution; NSJ Bioreagents, San Diego, CA, USA), mouse recombinant monoclonal anti-EPCAM (1:500 dilution; BioLegend, San Diego, CA, USA), and mouse recombinant monoclonal anti-pancytokeratin (cocktail of epithelial cytokeratins CK 7, 8, 9, 17, 18, and 19; 1:500 dilution; NSJ Bioreagents) antibodies. The slides were dewaxed in an oven at $60{ }^{\circ} \mathrm{C}$ for $30 \mathrm{~min}$ and by an immediate washing in baths with detergent (EZ PREP $1 \times$ ) for 5 min to remove the remaining paraffin from the slides. For antigen recovery, samples were subsequently immersed in a $\mathrm{pH} 6$ citrate buffer (trisodium citrate dehydrate; Sigma-Aldrich, St. Louis, MO, USA) preheated for $5 \mathrm{~min}$ in a microwave oven. Then slides were heated for $10 \mathrm{~min}$ in a microwave pressure cooker (Nordic Ware, St. Louis Park, MN, USA) until temperature of $120^{\circ} \mathrm{C}$ was reached. Once the samples cooled down to room temperature, they were immersed in distilled water for $10 \mathrm{~min}$ and then immediately immersed in phosphate-buffered saline (PBS) (HyClone, Logan, UT, USA) with $10 \%$ Tween (Sigma-Aldrich) for $5 \mathrm{~min}$. Subsequently, endogenous peroxidase and proteins (background staining) were blocked using Bloxall (Vector Laboratories, Burlingame, CA, USA) for $20 \mathrm{~min}$ and $2.5 \%$ normal horse serum (Vector Laboratories) for $20 \mathrm{~min}$ at room temperature. After carefully removing the horse serum, the samples were incubated with primary antibodies along with antibody diluent (Leyca Biosystems, Wetzlar, Germany) at $4{ }^{\circ} \mathrm{C}$ overnight. The following day, primary antibodies were detected using the ImmPRESS ${ }^{\circledR}$ horseradish peroxidase (HRP) horse anti-goat, anti-rabbit, and anti-mouse IgG polymer detection kits (Vector Laboratories), for anti- $\alpha$ SMA, anti-fibronectin, and anti-vimentin, anti-EPCAM, and anti-pan-cytokeratin antibodies, respectively. All secondary antibodies were incubated for $30 \mathrm{~min}$ at room temperature. Once the secondary antibodies were washed, slides were covered in chromogen diaminobenzidine (Abcam) for $5 \mathrm{~min}$, immediately washed twice in PBS (HyClone) to inhibit the chromogen reaction, and then immersed in distilled water for $5 \mathrm{~min}$. Next, the slides were covered with Harris hematoxylin (Golden Bell, Guadalajara, Mexico) for $30 \mathrm{~s}$ and immediately washed with filtered water for 5 $\mathrm{min}$. The slides were then treated with lithium carbonate (Golden Bell) and washed again in filtered water for $5 \mathrm{~min}$. Isopropyl alcohol (ICR, Mexico City, Mexico) was used to dry the slides, and the samples were immediately mounted using rapid mounting medium (Sigma-Aldrich). Duplicates were made for each type of immunohistochemistry staining. Colon tissue from a human autopsy was used as a control for immunohistochemical markers in all stainings. In fact, epithelial and stromal cells of this tissue were used as negative controls for desmoplastic stroma and EMT markers, respectively.

\section{Immunohistochemistry evaluation procedure}

To avoid subjectivity in the immunohistochemistry staining evaluation, computer-assisted quantitative image analysis was performed using Aperio ePathology Solutions software (Leica Biosystems Imaging Inc., Wetzlar, Germany). Digital images of the slides were acquired using a ScanScope digital scanner (Aperio ePathology Solutions). The acquired images were observed and organized using the ImageScope viewer. The Aperio Spectrum/eSlide manager system (Aperio ePathology Solutions) was used to quantify the DS and EMT markers. The percentage of marker expression in pixels (positive staining area) was calculated using the V9 positive pixel count algorithm according to the immunohistochemical analysis of each marker. ${ }^{55}$ A PDAC expert pathologist selected the tumor core (total expression for each antibody) and the areas in the TMA corresponding to desmoplastic stromal tissue and tumor tissue. During the selection of areas, artifacts (tissue folds) that could affect the analysis were ignored. The Aperio software was used to calculate the percentage of expression in pixels, which was classified as moderate pixel expression [number of positive pixels $(\mathrm{Np})$ ], strong pixel expression [number of strong positive pixels (Nsp)], negative pixel expression [number of negative pixels $(\mathrm{Nn})$ ], and total pixel expression, [number of total pixels, positive + negative $(\mathrm{Nt})$ ). The following formula was used to calculate the percentage of expression in pixels: $\mathrm{Np}+\mathrm{Nsp} / \mathrm{Nt}$ $\times 100 .{ }^{55,56}$ Values for each tumor core and each tissue area were averaged according to the number of samples obtained per patient (triplicates). The resulting quantification was the net number for each sample (only the cores containing at least $50 \%$ of the sample were included in the analysis).

\section{Statistical analysis}

To visually compare the patterns of protein expression percentages between each clinical stage, differentiation grade, and survival, a heatmap of the Z-score was constructed according to previous studies. ${ }^{52,57}$ We made a heatmap based on a hierarchical grouping of the expression levels of each marker using a data normalization of the $\mathrm{Z}$ distribution (difference between baseline minus mean between standard deviation) then we order it by clinical stage, degree of differentiation and survival. The values were classified according to the band of the $\mathrm{Z}$ score as high expression (pink to red colors) if the data for each marker were above average, and low expression (light to dark blue colors) if the data for each marker were below average. The average data for each antibody were marked in white, whereas data for each antibody that could not be obtained were marked in black.

SPSS ver. 21 software (IBM, Armonk, NY, USA) was used to perform statistical analyses. Chi square test was used to determine the association between clinical stage, degree of differentiation, survival and resectability (categorical parameters) and the expression of markers in tumor cores in desmoplastic stromal tissues and tumor tissues were classified as high and low expression, and the average of each marker was considered the cut-off point. Marker data was classified as categorical parameters: 0 (low expression) and 1 (high expression). Data were considered significant when the $\mathrm{p}$ value was $<0.05$.

Overall survival was calculated as the time elapsed from the date of pathology diagnosis to the date of death or the date of the last follow-up if the patient was still alive. Kaplan-Meier analysis was used to evaluate the relationship between the expression level of the studied proteins and the general survival time. The samples were classified as high and low expression, and the average of each marker was considered the cut-off point. In addition to the global expression of each protein, data were subclassified considering the clinical stage of the patients and the degree of differentiation. The Log-rank test was used to assess the importance of differences in 
survival curves between samples with high or low expression of marker, ${ }^{58}$ using the data analysis software Origin 8 (OriginLab, Northampton, MA, USA). Data were considered significant when the p-value was $<0.05$.

\section{Results}

\section{Clinicopathological findings}

Clinicopathological data were retrieved from a database generated from clinical records of the Medical Archive department of the Oncology Hospital. TNM stage was determined according to the TNM classification, which was determined from preoperative imaging findings, intraoperative findings, and postoperative pathological findings. ${ }^{5}$ Our study collected data on 15 male $(60 \%)$ and 10 female (40\%) PDAC patients, with a mean age at diagnosis of 63 years (ranging from 41 to 82 years). We found at the diagnostic time that most of the patients were classified in the advanced stage $\mathrm{T}$ and $\mathrm{N}$, however most patients at stage $\mathrm{M}$ were found to correspond to M0. Clinical stage II was predominant in about $56 \%$ of the cases, while clinical stages III and IV corresponded to the remaining $44 \%$. Regarding the degree of differentiation samples of most patients displayed a moderate degree of differentiation (64\%). Evolution time ranged from 0 to 32 months, the follow-up time of the first and the last medical consultation ranged from 1 to 33 months, and the follow-up time of the disease onset at the last visit ranged from 0 to 39 months. In our study cohort, the mean overall survival was 5 months (range, 1-31 months). All cases were positive for desmoplasia, and the predominant type of surgery was Whipple procedure $(44 \%)$. Clinicopathological data of the patients with PDAC are outlined in Table 1.

\section{Expression of DS and EMT markers in PDAC samples}

In this study, 75 samples from a cohort of 25 patients diagnosed with PDAC were analyzed to detect DS ( $\alpha$-SMA, fibronectin, and vimentin) and EMT (EPCAM, pan-cytokeratin, and vimentin) markers. These markers were selected because their expression has been suggested to affect the prognosis of patients with PDAC. ${ }^{17}$

The study markers were validated using a control sample of colon tissue (Figure 1). The $\alpha$-SMA (Figure 1A), fibronectin (Figure 1E), and vimentin (Figure 1I) markers were expressed in the cytoplasm of stromal cells surrounding the colon epithelial glands (periglandular staining). The EPCAM (Figure 1M) and pancytokeratin (Figure 1Q) markers were expressed in the membrane and cytoplasm of colon epithelial cells (glandular staining), respectively. Tumor samples showed regions of DS in stromal tissue (Table 1). We did not find samples of clinical stage I in our study (Table 1); thus, the expression of DS markers was evaluated in stages II, III, and IV. The stromal markers $\alpha$-SMA, fibronectin, and vimentin were located around the glands and tumor cells (perilesional staining) in the tumors (Figure 1, B-D; F-H; and J-L). Positive $\alpha$-SMA staining was observed in 22 cases $(88 \%), 13$ of which were at clinical stage II (52\%, Figure 1B), 4 at clinical stage III (16\%, Figure 1C), and 5 at clinical stage IV (20\%, Figure 1D). Positive $\alpha$-SMA staining was found in $56 \%$ of cases with a moderate differentiation grade (Figure 1C) and in $32 \%$ of cases with a poor differentiation grade (Figures 1B and 1D). Positive fibronectin staining was observed in 21 cases (84\%), 13 of which were at clinical stage II (52\%, Figure 1F), 4 at clinical stage III $(16 \%$, Figure $1 \mathrm{G})$, and 4 at clinical stage IV $(16 \%$, Figure $1 \mathrm{H})$. Positive fibronectin staining was observed in $52 \%$ of cases with a moderate differentiation grade (Figure $1 \mathrm{G}$ ) and in 32\% of cases with a poor differentiation grade (Figures 1 F,H). The expression of epithelial markers of EMT was also evaluated in tumor tissue (vimentin, pan-cytokeratin and EPCAM; Figure $1 \mathrm{~J}-\mathrm{L}, \mathrm{N}-\mathrm{P}, \mathrm{R}-\mathrm{T}$ ). Positive vimentin staining was found in 24 cases $(96 \%), 13$ of which were at clinical stage II (52\%), 5 at clinical stage III (20\%), and 6 at clinical stage IV (24\%). Positive vimentin staining was found in $60 \%$ of cases with a moderate differentiation grade, but the staining was weak in most cases (data not shown) and negative in tumor glands (Figures $1 \mathrm{~J}, \mathrm{~K}$ ). Positive vimentin staining was found in $36 \%$ of cases with a poor differentiation grade (Figure $1 \mathrm{~L})$. Both in DS and EMT, vimentin expression was observed in the same percentage for clinical stage and degree of differentiation.

Table 1. Clinicopathological features of a retrospective cohort of 25 patients with PDAC.

\section{Parameter} Total $(n=25)$

Sex $25(100 \%)$

Male/female $15 / 10(60 \% / 40 \%)$

Verage age at the time of surgery rank $63(41-82)$

Stage T $25(100 \%)$

T2 $4(16 \%)$

T3

T4 $12(48 \%)$ Stage N $9(36 \%)$ NO NO N1 $25(100 \%)$ No

Stage $4(16 \%)$ $14(56 \%)$ tage M $7(28 \%)$ pMl AJCC stage $25(100 \%)$ II III IV

Degree of differentiation $\quad 25(100 \%)$ $19(76 \%)$

M1 $6(24 \%)$

II $14(56 \%)$

II $20 \%)$

Well differentiated $0(0 \%)$

Moderadately differentiated $\quad 16(64 \%)$

Poorly differentiated $9(36 \%)$

Evolution time $24(96 \%)$

Median evolution time in months (rank) $1(0-32)$

Follow-up time of the first and the last medical consultation $21(84 \%)$

Median follow-up time of the first and the last medical consultation (rank) 8 (1-33) Follow-up time of the disease onset at the last visit $24(96 \%)$

Median follow-up time of the disease onset at the last visit (rank) $12(0-39)$

Survival $22(88 \%)$

Median survival in months (rank) $5(1-31)$

Desmoplasia $25(100 \%)$

Type of surgery $20(80 \%)$

Whipple $11(44 \%)$

Lape $3(12 \%)$

Subtotal pancreatectomy with splenectomy $\quad 1(4 \%)$

Multistructural resection $1(4 \%)$

Whipple and lape $2(8 \%)$

Pancreatectomy $2(8 \%)$

AJCC, American Joint Committee on Cancer. 
Positive pan-cytokeratin staining was observed in 24 cases (96\%), 14 of which were at clinical stage II (56\%, Figure 1N), 5 at clinical stage III (20\%, Figure 1O), and 5 at clinical stage IV $(20 \%$, Figure 1P). Positive pan-cytokeratin staining was found in $60 \%$ of cases with a moderate differentiation grade (Figure 10) and in $36 \%$ of cases with a poor differentiation grade (Figures 1 N,P). Positive EPCAM staining was found in all 25 cases (100\%), 14 of which were at clinical stage II (56\%, Figure 1R), 5 at clinical stage III (20\%, Figure 1S), and 6 at clinical stage IV (24\%, Figure $1 \mathrm{~T})$. EPCAM-positive staining was found in $64 \%$ of cases with a moderate differentiation grade, marking tumor glands (Figure 1S), and in $36 \%$ of cases with a poor differentiation grade, showing a diffuse staining of the marker (Figure 1R). However, in some cases, EPCAM-negative areas were also observed in disaggregated tumor cells (Figure 1T)

The vimentin (Figure 1L), pan-cytokeratin (Figure 1P), and EPCAM (Figure 1T) photomicrographs derived from a serial case with a poor differentiation grade show positive staining for vimentin and pan-cytokeratin but not for EPCAM. All markers of
DS and EMT were positive in our cohort of study.

\section{Clinicopathological variables and their association with DS and EMT markers}

Once the expression of the markers was determined, we decided to analyze if there was an association between the DS and EMT with clinicopathological variables. For this purpose, we first identified if there were differences in the expression levels of each marker. For this evaluation, a visual multivariate analysis was performed, and a heatmap was produced for the z-scores (Figure 2). This method evidenced the expression heterogeneity of each study marker for the 25 cases of our cohort included AJCC stage, degree of differentiation and survival (Figure 2). For this reason, each marker was separated using its average expression in pixels as a reference and we classified each marker as either low or high expression in pixels. High expression of vimentin in tumor core was associated with clinical stages III and IV $(p=0.041)$. High expression of $\alpha$-SMA in tumor core $(p=0.033)$, EPCAM $(p=0.030)$ and pan-cytokeratin $(\mathrm{p}=0.050)$ in tumor tissue were associated
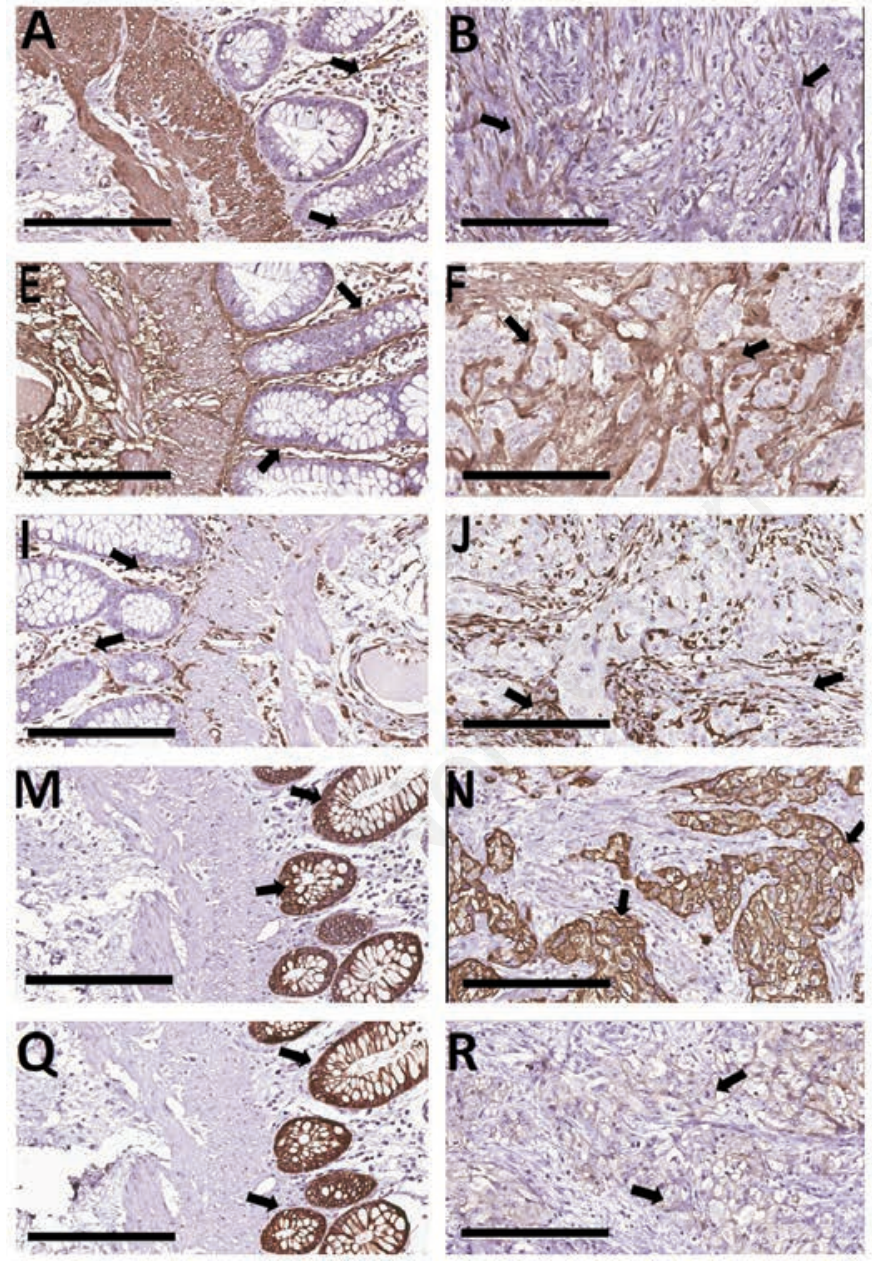
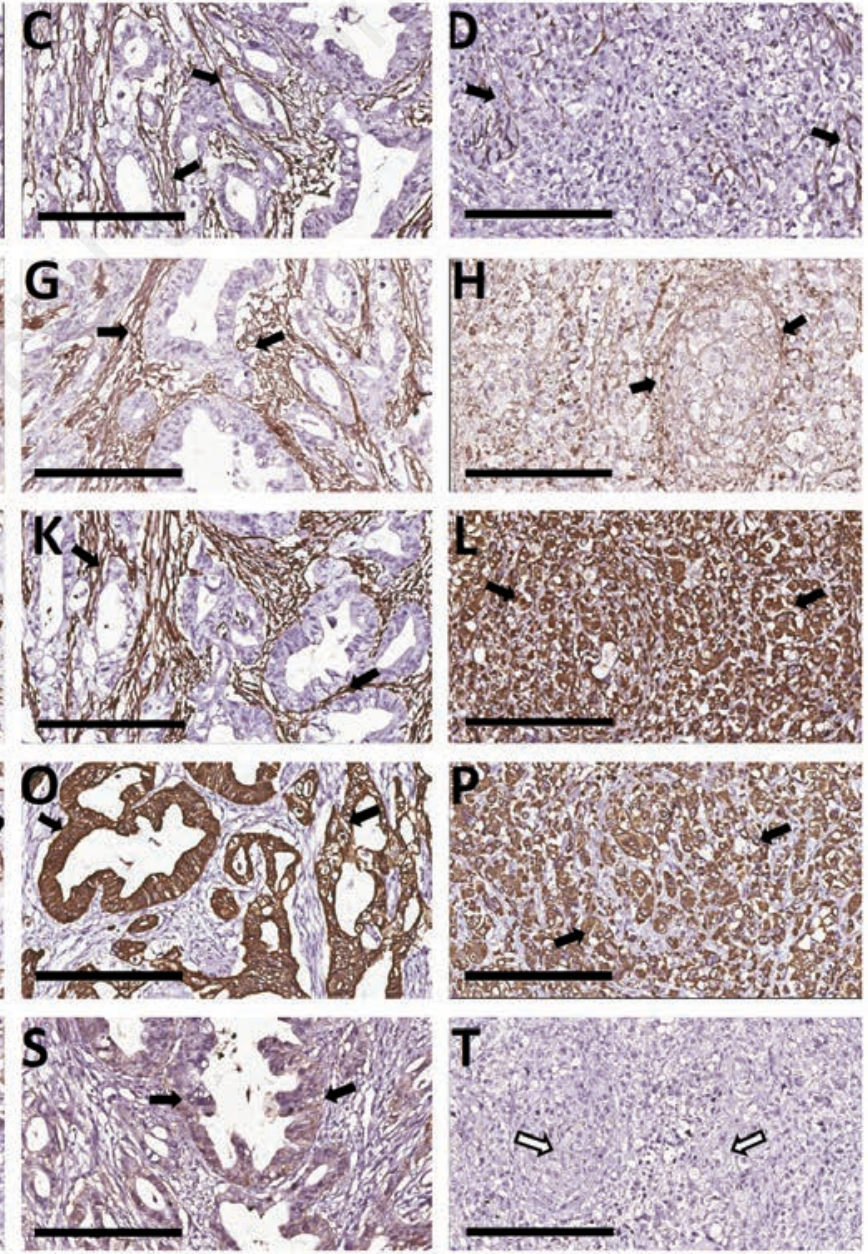

Figure 1. Immunohistochemical analysis of DS and EMT markers in control colon and PDAC tissues. Representative microphotographs of control colon tissues (A-Q). Periglandular staining of $\alpha$-SMA-positive stromal cells (A). Periglandular staining of fibronectin-positive stromal cells (E). Periglandular staining of vimentin-positive stromal cells (I). Epithelial glands staining positive for EPCAM (M). Epithelial glands staining positive for pan-cytokeratin (Q). Black arrows indicate positive staining in stroma (A-I); Black arrows indicate positive staining in glands $(M, Q)$. Representative cases of samples from patients with PDAC (B-T). Clinical stages II (B-R), III (C-S), and IV (DT). Black arrows indicate perilesional $\alpha-S M A(B-D)$, fibronectin $(F-H)$, and vimentin $(\mathrm{J}-\mathrm{K})$ staining in tumor stroma. Black arrows indicate vimentin (L), pan-cytokeratin (N-P), and EPCAM (R-S) staining in tumor cells. White arrows indicate negative EPCAM staining in tumor cells (T). All stains were counterstained using hematoxylin. Magnification: 20x. Scale bars: $200 \mu \mathrm{m}$. 
with a lower survival corresponding to less than 5 months. Low expression of fibronectin in tumor core was associated with a positive resectability $(p=0.026$ ). High expression of $\alpha$-SMA in stromal tissue was associated with a poor differentiation grade $(\mathrm{p}=0.035)$. These results are summarized in Tables 2 and 3.

\section{High expression levels of DS and EMT markers are associated with decreased survival as a function of clin- ical stage and degree of differentiation}

We performed survival analysis using the current prognostic stratification (Figure $3 \mathrm{~A}, \mathrm{~B}$ ) and found no statistically significant association between survival and clinical stage $(p=0.54)$ and between survival and degree of differentiation $(\mathrm{p}=0.40)$. Subsequently, we decided to identify subgroups for predicting a lower survival rate for different clinical stages and degree of differentiation according to the expression of DS and EMT markers in tumor core, stromal and tumor tissue. Based on our previous multivariate analysis used with clinicopathological variables, we classified each marker as displaying either low or high expression in color-coded pixels for each clinical stage and degree of differentiation. High expression levels of $\alpha$-SMA ( $\mathrm{p}=0.03618)$ at clinical stage II and fibronectin ( $p=0.01554)$ at clinical stages III and IV in stromal tissue were associated with lower survival (Figure 4 A,B). High expression of vimentin at clinical stages II ( $\mathrm{p}=0.00683)$, III and IV $(p=0.03735)$ in stromal tissue was associated with lower survival
(Figure 4 C,D). High expression of pan-cytokeratin $(\mathrm{p}=0.0393)$ at clinical stage II was associated with lower survival (Figure 4E). High expression of pan-cytokeratin $(p=0.00014)$ in tumor tissue and vimentin $(\mathrm{p}=0.03735)$ in tumor core were associated with lower survival at clinical stages III and IV (Figure 4 F,G). High expression levels of fibronectin and $\alpha$-SMA were associated with a lower survival for moderate $(\alpha$-SMA $p=0.02$ ) and poor (fibronectin $\mathrm{p}=0.01$ ) differentiation grades in stromal tissue (Figure $4 \mathrm{H}, \mathrm{I})$. High expression levels of vimentin $(\mathrm{p}=0.012)$ in stromal tissue were associated with lower survival for moderate differentiation grade (Figure 4J). High expression levels of pan-cytokeratin and EPCAM were associated with a lower survival for poor differentiation grade (pan-cytokeratin $\mathrm{p}=0.00185$, EPCAM $\mathrm{p}=0.000085$ ) in tumor tissue (Figure $4 \mathrm{~K}, \mathrm{~L}$ ). High expression levels of pan-cytokeratin $(p=0.00977)$ in tumor core were associated with lower survival for moderate differentiation grade (Figure 4M).

\section{Discussion}

In this study, we found that high expression levels of DS markers, such as $\alpha$-SMA, fibronectin, and vimentin, were associated with lower patient survival at intermediate and advanced clinical stages, as well as with poorly and moderately differentiated grades. Interestingly, the same patterns were observed when analyzing the

Table 2. Association of clinicopathological features with markers on tumor core.

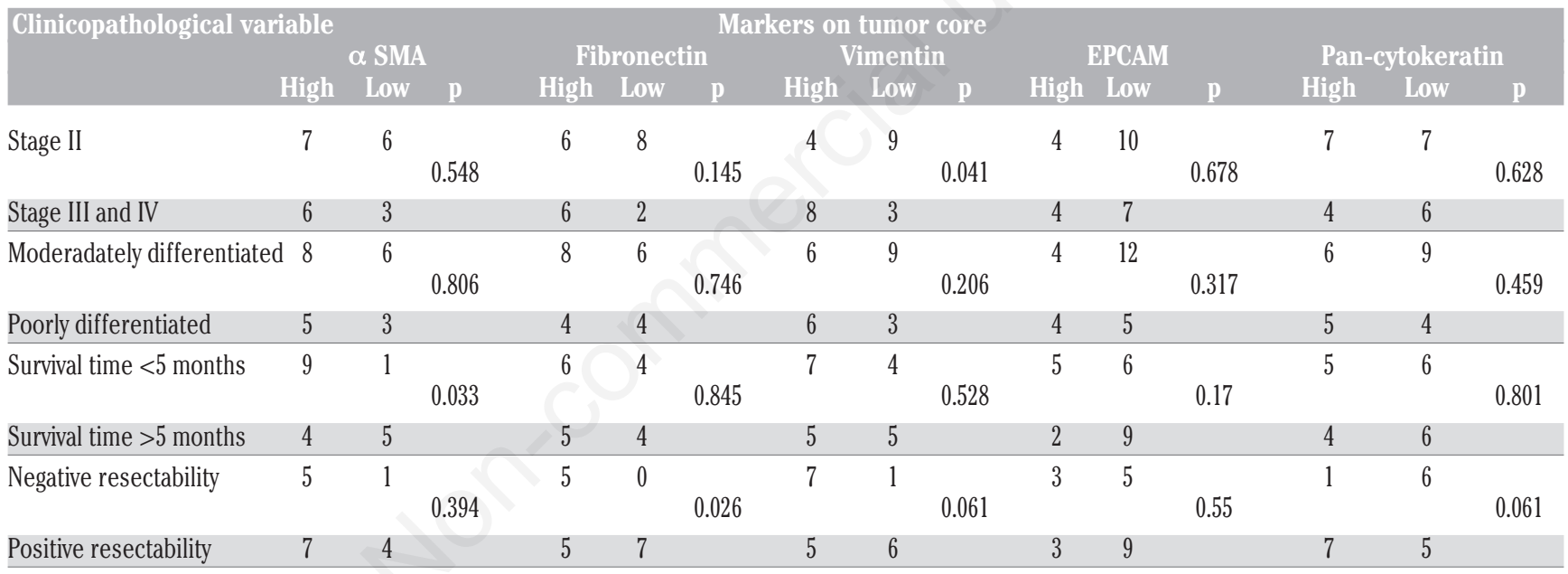

Table 3. Association of clinicopathological features with markers on stromal and tumor tissue.

\begin{tabular}{|c|c|c|c|c|c|c|c|c|c|c|c|c|c|c|c|c|c|c|}
\hline \multirow{2}{*}{\multicolumn{4}{|c|}{$\begin{array}{l}\text { Clinicopathological variable } \\
\qquad \begin{array}{r}\alpha \text { SMA } \\
\text { (stromal) } \\
\text { High Low p }\end{array}\end{array}$}} & \multicolumn{9}{|c|}{ Markers on stromal and tumor tissue } & \multirow{2}{*}{\multicolumn{3}{|c|}{$\begin{array}{l}\text { Pan-cytokeratin } \\
\text { (tumor) }\end{array}$}} & \multirow{2}{*}{\multicolumn{3}{|c|}{$\begin{array}{l}\text { Vimentin } \\
\text { (tumor) }\end{array}$}} \\
\hline & & & & \multicolumn{3}{|c|}{$\begin{array}{l}\text { Fibronectin } \\
\text { (stromal) }\end{array}$} & \multicolumn{3}{|c|}{$\begin{array}{l}\text { Vimentin } \\
\text { (stromal) }\end{array}$} & \multicolumn{3}{|c|}{$\begin{array}{l}\text { EPCAM } \\
\text { (tumor) }\end{array}$} & & & & & & \\
\hline & & & & High & Low & p & High & Low & p & High & Low & p & High & Low & p & High & Low & p \\
\hline Stage II & 6 & 7 & 0.937 & 7 & 6 & 0.466 & 6 & 7 & 0.188 & 5 & 9 & 0.622 & 7 & 7 & 1 & 3 & 10 & 0.247 \\
\hline Stage III and IV & 4 & 5 & & 3 & 5 & & 8 & 3 & & 5 & 6 & & 5 & 5 & & 5 & 6 & \\
\hline Moderadately differentiated & d 4 & 10 & 0.035 & 5 & 8 & 0.284 & 7 & 8 & 0.134 & 6 & 10 & 0.734 & 7 & 8 & 0.673 & 3 & 12 & 0.074 \\
\hline Poorly differentiated & 6 & 2 & & 5 & 3 & & 7 & 2 & & 4 & 5 & & 5 & 4 & & 5 & 4 & \\
\hline Survival time $<5$ months & 6 & 4 & 0.498 & 5 & 5 & 0.28 & 8 & 3 & 0.284 & 7 & 4 & 0.03 & 8 & 3 & 0.05 & 3 & 8 & 0.284 \\
\hline Survival time $>5$ months & 4 & 5 & & 2 & 6 & & 5 & 5 & & 2 & 9 & & 3 & 7 & & 5 & 5 & \\
\hline Negative resectability & 2 & 4 & 0.232 & 2 & 3 & 0.889 & 5 & 3 & 0.96 & 5 & 3 & 0.094 & 5 & 2 & 0.109 & 4 & 4 & 0.552 \\
\hline ositive resectability & 7 & 4 & & 4 & 7 & & 7 & 4 & & 3 & 9 & & 4 & 8 & & 4 & 7 & \\
\hline
\end{tabular}


EMT markers vimentin, pan-cytokeratin, and EPCAM. To our knowledge, this is the first study to establish an association between the expression of DS and EMT markers and survival by clinical stage and degree of differentiation, suggesting that their evaluation might be of help in estimating the survival time in patients with PDAC.

In our study cohort, a key issue is that vimentin has started to be evaluated as a DS marker in patients with PDAC, ${ }^{59}$ because it has mainly been described as an EMT marker; ${ }^{48,60,61}$ however, we detected its expression in stromal tissue surrounding tumor glands, in line with previous observations. ${ }^{62}$ In contrast to other studies which have reported the expression of pan-cytokeratin in tumor buddings, ${ }^{46,63}$ we detected pan-cytokeratin expression in glands and scattered tumor cells surrounded by DS in PDAC samples. Also, we observed a weak or even null EPCAM expression in areas of disaggregated tumor cells, which could also be found in EMT. Similar results have been described in in vitro cultures of breast tumor cells, which also showed decreased EPCAM expression associated with the EMT process. ${ }^{34}$ In our study, vimentin was mainly expressed in disaggregated tumor cells. Therefore, the

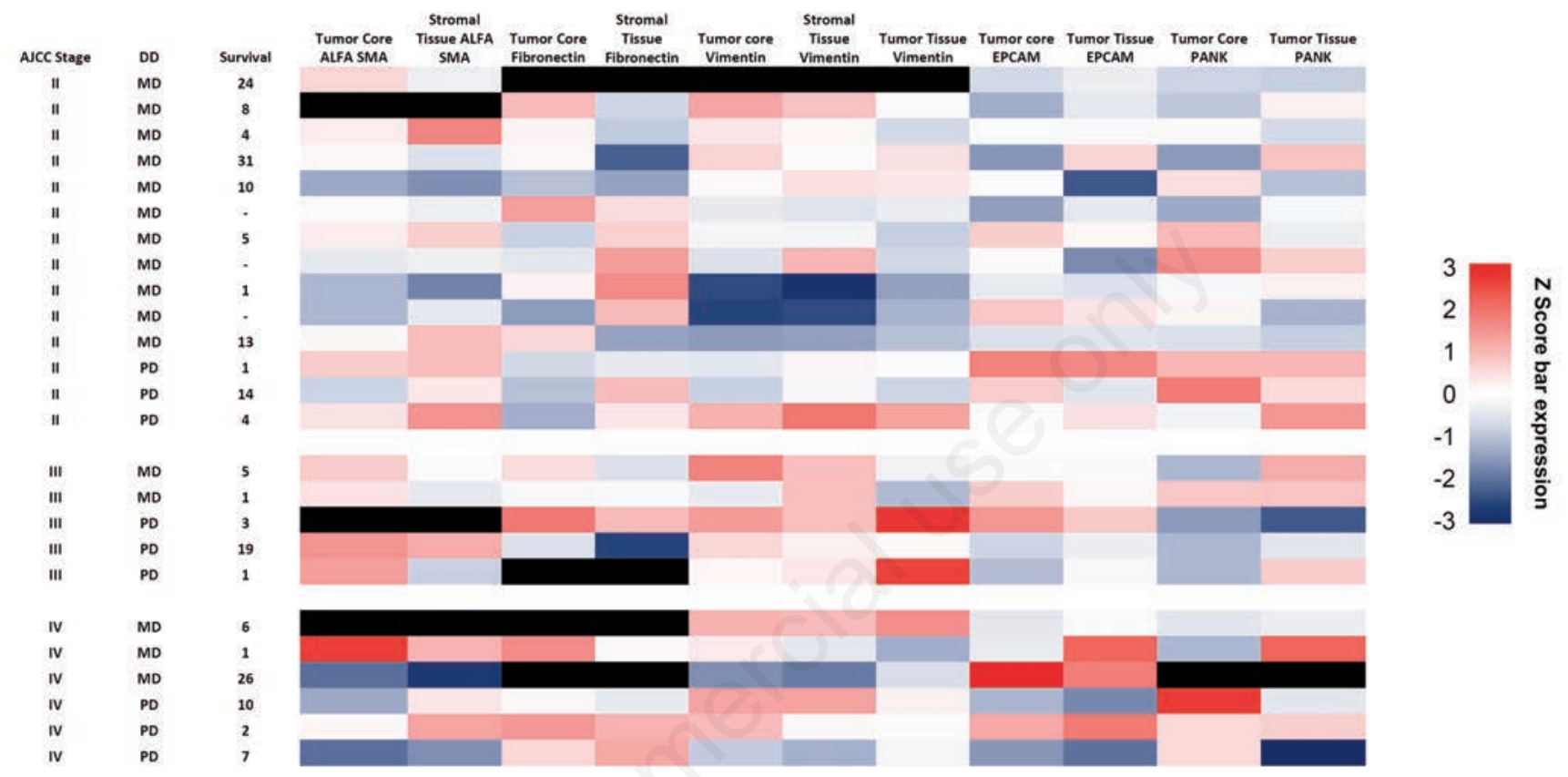

Figure 2. Heatmap of the Z-scores. Low staining levels are indicated by values ranging from -1 to -3 (blue color). High staining levels are indicated by values ranging from 1 to 3 (red color). Cells without values are indicated in black, and those that correspond to the average expression levels are indicated in white. Degree of differentiation (DD): moderately differentiated (MD) and poorly differentiated (PD). Survival is indicated in months.
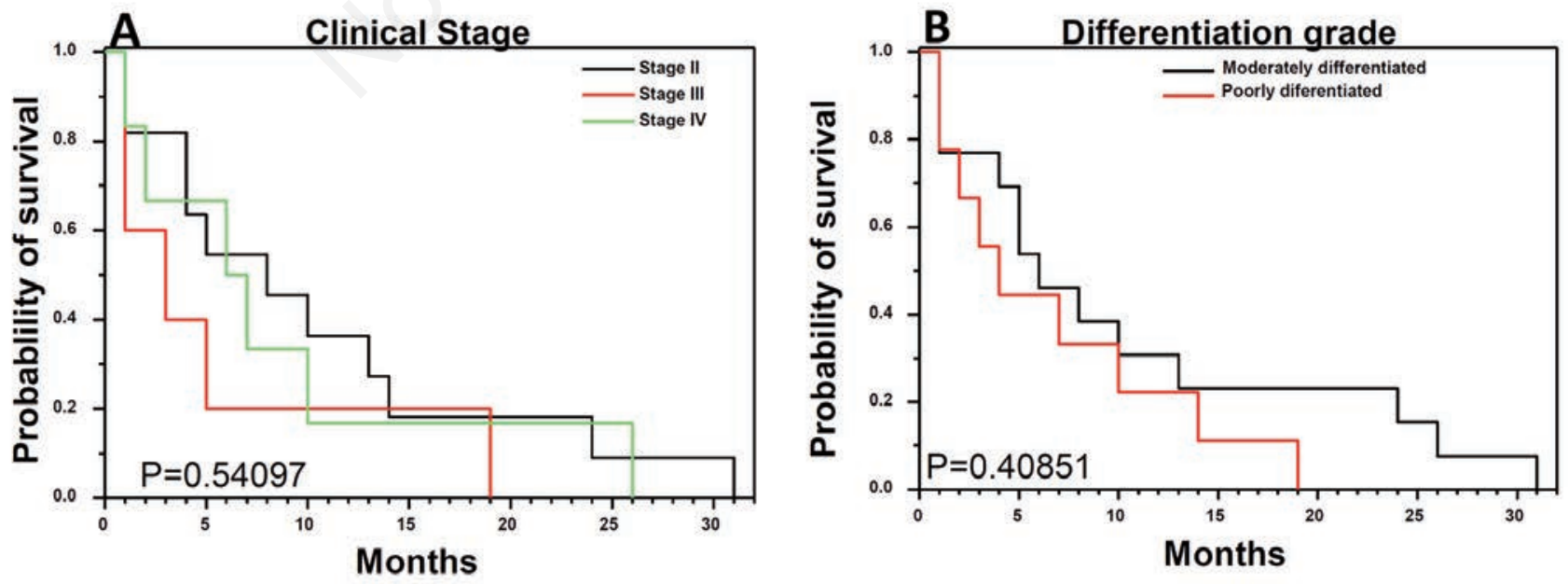

Figure 3. Current prognostic stratification and survival of patients with PDAC. Kaplan-Meier analysis of the overall survival of patients and clinical stage. Samples are marked in black for clinical stage II, in red for clinical stage III, and in green for clinical stage IV (A). Kaplan-Meier analysis of the overall survival of patients and differentiation grade. Samples with a moderate differentiation grade are marked in black, and samples with a poor differentiation grade are marked in red (B). 


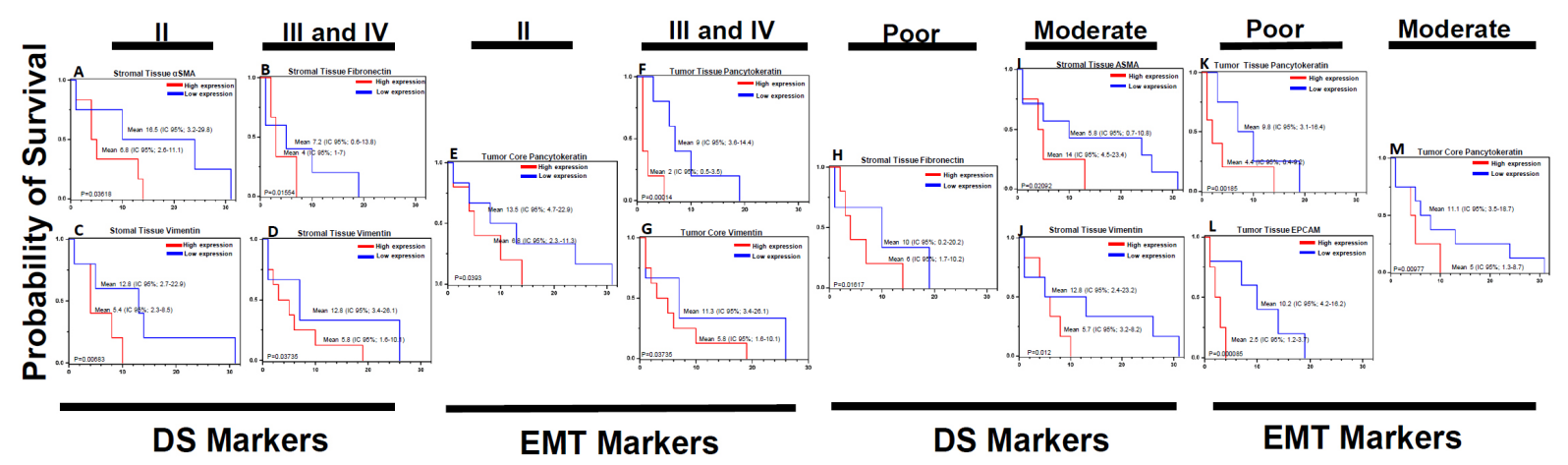

-Time in months-

Figure 4. Association of DS markers and EMT with survival by clinical stage and degree of differentiation in PDAC. The expression of DS and EMT markers was quantified and associated with the overall survival of patients in a Kaplan-Meier analysis by clinical stage (A-G) and degree of differentiation (H-M). Survival analysis of $\alpha-S M A(n=10 ; A)$, vimentin $(n=10$; C) in stromal tissue and pancytokeratin in tumor core $(n=11 ; E)$ were identified in clinical stage II. Survival analysis of fibronectin $(n=8 ; B)$, vimentin in stromal tissue $(n=11 ; D)$, pan-cytokeratin in tumor tissue $(n=10 ; F)$ and vimentin in tumor core $(n=11$; $G)$ were identified in clinical stages III and IV. Survival analysis of $\alpha$-SMA $(n=13 ; I)$, vimentin $(n=15 ; J)$ in stromal tissue and pan-cytokeratin in tumor core $(n=14 ; M)$ were identified in moderate degrees of differentiation. Survival analysis of fibronectin $(n=8 ; H)$ in stromal tissue, pan-cytokeratin ( $n=9$; $K$ ) and $\operatorname{EPCAM}(n=9 ; \mathrm{L})$ in tumor tissue were identified in poor degrees of differentiation. For clinical stage, samples with low expression are marked in blue, and samples with high expression are marked in red. Similarly, mean survival values and $95 \%$ confidence intervals (CI) are shown, and the p-values of the Log-rank test are shown.

close contact with DS suggests that in PDAC, these cells could be more easily released into the circulation in groups or individually. ${ }^{36,63,64}$ This is the first study to jointly assess the expression of two epithelial markers (pan-cytokeratin and EPCAM) and one mesenchymal marker (vimentin) by immunohistochemistry in PDAC and to report the association between high expression of vimentin with advanced clinical stage (III and IV). This dual interaction could explain why these types of tumors are more aggressive at advanced stages. Furthermore, the association of pan-cytokeratin with lower survival rates has never been reported, so far studies have only evaluated the expression of this marker as an identifier of tumor budding, which in turn is associated with a lower survival rate. ${ }^{46}$ On the other hand, $\alpha$-SMA has never been reported associated with a less degree of differentiation which involves the importance of DS in the development of more aggressive tumors and EMT, as reported in in vitro studies. ${ }^{14,20,23,29,31}$ The low expression of fibronectin was associated with a positive resectability, which implies that a minor quantity of DS could facilitate surgical intervention, because this stromal marker is part of the fibrotic tissue secreted by stromal cells that promote the development of the DS, ${ }^{16,39,65}$ which occupies more than $80 \%$ of the tumor volume. ${ }^{17}$ This association is reported for the first time.

According to the current prognostic stratification based on clinical stage (TNM) and degree of differentiation $(G)$, in our study, these parameters were not associated with the survival of patients diagnosed with PDAC, in line with other published studies, albeit with a higher number of participants; ${ }^{65}$ yet these studies contrast with others that incorporated a higher number of patients. ${ }^{4,6}$ Despite these differences, in each clinical stage and in even advanced stages, heterogeneity has been observed in the survival time of patients, which is not explained by the current prognostic stratification. ${ }^{41,42}$

When choosing a panel of DS and EMT antibodies for each patient, we found in our study cohort that all markers were heterogeneously expressed, as shown by the heatmap, thus indicating the presence of high- and low-expression subgroups. Our quantitative analysis allowed us to observe that the higher expression of all markers defined a shorter survival for each clinical stage and degree of differentiation, in contrast to other studies in which these markers were individually evaluated in terms of overall survival of patients with PDAC. ${ }^{44,45,48,65}$ Some preliminary findings from a semi-quantitative analysis performed in patients with PDAC indicates that EMT markers may be associated with a shorter survival in clinical stages III and IV ${ }^{40,45}$ and stromal markers could be associated with a shorter survival in clinical stages II; ${ }^{44}$ in fact in our cohort of Mexican patients with this subtype of pancreatic cancer this behavior is observed. However, when classifying clinical stages and differentiation grades by subgroups, our study cohort was reduced; hence, further studies should be conducted using an increased sample size. Furthermore, markers such as $\mathrm{T} \beta_{4}$, which has been suggested plays an important role in the process of EMT in colorectal cancer ${ }^{38}$ and the roles of different subsets of CAFs within the stroma ${ }^{12}$ could also be evaluated.

In conclusion, we suggest that including DS and EMT markers in prognostic stratification may improve our understanding of different survival times of patients with PDAC, regardless of the clinical stage and degree of differentiation at which they are diagnosed, thereby improving current prognostic stratification.

\section{Acknowledgements}

The first author thanks to the Programa de Doctorado de Ciencias Biomédicas, Universidad Nacional Autónoma de México, UNAM, and received fellowship 432566 from CONACyT, México and Instituto Mexicano del Seguro Social (IMSS) (99097191). We gratefully acknowledge the excellent technical assistance of Galo Abad Méndez Matias, Daniel Enríquez Ramírez, Miguel Ángel Sandoval Hernández, Alam Palma Guzmán, Luis Edgar Arias Martínez and Dr. Eugenia Flores-Figueroa. 


\section{References}

1. International Agency for Research on Cancer. Cancer today. Accessed: 6 June 2019. Available from: http://gco. iarc.fr/today/home

2. Rawla P, Sunkara T, Gaduputi V. Epidemiology of pancreatic cancer: Global trends, etiology and risk factors. World J Oncol 2019; 10:10-27.

3. Hidalgo M, Cascinu S, Kleeff J, Labianca R, Löhr J-M, Neoptolemos J, et al. Addressing the challenges of pancreatic cancer: future directions for improving outcomes. Pancreatology 2015;15:8-18

4. Bilimoria KY, Bentrem DJ, Ko CY, Ritchey J, Stewart AK, Winchester DP, et al. Validation of the 6th edition AJCC pancreatic cancer staging system. Cancer 2007;110:738-44.

5. Amin MB, Edge S, Greene F, Byrd DR, Brookland RK, Washington MK, et al., Manual de estadificación del cáncer del AJCC. Springer; 2017.

6. Wasif N, Ko CY, Farrell J, Wainberg Z, Hines OJ, Reber H, et al. Impact of tumor grade on prognosis in pancreatic cancer: should we include grade in AJCC staging? Ann Surg Oncol 2010; 17:2312-20.

7. Ren H, Wu C-R, Qiu G-T, Zhang L-P, Aimaiti S, Wang C-F. Equipping the American Joint Committee on cancer staging for resectable pancreatic ductal adenocarcinoma with tumor grade: A novel staging system. J Oncol 2020;2020:9093729.

8. Rochefort MM, Ankeny JS, Kadera BE, Donald GW, Isacoff $\mathrm{W}$, Wainberg ZA, et al. Impact of tumor grade on pancreatic cancer prognosis: validation of a novel TNMG staging system. Ann Surg Oncol 2013;20:4322-9.

9. Hlavsa J, Cecka F, Zaruba P, Zajak J, Gurlich R, Strnad R, et al. Tumor grade as significant prognostic factor in pancreatic cancer: validation of a novel TNMG staging system. Neoplasma 2018;65:637-43.

10. Winter JM, Cameron JL, Campbell KA, Arnold MA, Chang DC, Coleman J, et al. 1423 pancreaticoduodenectomies for pancreatic cancer: A single-institution experience. J Gastrointest Surg 2006;10:1199-210.

11. Vincent A, Herman J, Schulick R, Hruban RH, Goggins M. Pancreatic cancer. Lancet 2004;363:1049-57.

12. Sperb N, Tsesmelis M, Wirth T. Crosstalk between tumor and stromal cells in pancreatic ductal adenocarcinoma. Int $\mathrm{J}$ Mol Sci 2020;21:e5486.

13. Peran I, Madhavan S, Byers SW, McCoy MD. Curation of the pancreatic ductal adenocarcinoma subset of the cancer genome atlas is essential for accurate conclusions about survival-related molecular mechanisms. Clin Cancer Res 2018;24:3813-9.

14. Waghray M, Yalamanchili M, Dziubinski M, Zeinali M, Erkkinen M, Yang H, et al. GM-CSF mediates mesenchymalepithelial cross-talk in pancreatic cancer. Cancer Discov 2016;6:886-99.

15. Erkan M, Kleeff J, Gorbachevski A, Reiser C, Mitkus T, Esposito I, et al. Periostin creates a tumor-supportive microenvironment in the pancreas by sustaining fibrogenic stellate cell activity. Gastroenterology 2007;132:1447-64.

16. Omary MB, Lugea A, Lowe AW, Pandol SJ. The pancreatic stellate cell: a star on the rise in pancreatic diseases. J Clin Invest 2007; 117:50-9.

17. Nielsen MFB, Mortensen MB, Detlefsen S. Key players in pancreatic cancer-stroma interaction: Cancer-associated fibroblasts, endothelial and inflammatory cells. World J Gastroenterol 2016;22:2678-700.

18. Bachem MG, Schneider E, Gross H, Weidenbach H, Schmid RM, Menke A, et al. Identification, culture, and characterization of pancreatic stellate cells in rats and humans.
Gastroenterology 1998;115:421-32.

19. Eguchi D, Ikenaga N, Ohuchida K, Kozono S, Cui L, Fujiwara $\mathrm{K}$, et al. Hypoxia enhances the interaction between pancreatic stellate cells and cancer cells via increased secretion of connective tissue growth factor. J Surg Res 2013;181:225-33.

20. Vonlaufen A, Joshi S, Qu C, Phillips PA, Xu Z, Parker NR, et al. Pancreatic stellate cells: partners in crime with pancreatic cancer cells. Cancer Res 2008;68:2085-93.

21. Kalli M, Papageorgis P, Gkretsi V, Stylianopoulos T. Solid stress facilitates fibroblasts activation to promote pancreatic cancer cell migration. Ann Biomed Eng 2018;46:657-69.

22. Sada M, Ohuchida K, Horioka K, Okumura T, Moriyama T, Miyasaka Y, et al. Hypoxic stellate cells of pancreatic cancer stroma regulate extracellular matrix fiber organization and cancer cell motility. Cancer Lett 2016;372:210-8.

23. Öhlund D, Handly-Santana A, Biffi G, Elyada E, Almeida AS, Ponz-Sarvise M, et al. Distinct populations of inflammatory fibroblasts and myofibroblasts in pancreatic cancer. J Exp Med 2017;214:579-96.

24. Li D, Qu C, Ning Z, Wang H, Zang K, Zhuang L, et al. Radiation promotes epithelial-to-mesenchymal transition and invasion of pancreatic cancer cell by activating carcinomaassociated fibroblasts. Am J Cancer Res 2016;6:2192-206.

25. Marrache F, Pendyala S, Bhagat G, Betz KS, Song Z, Wang TC. Role of bone marrow-derived cells in experimental chronic pancreatitis. Gut 2008;57:1113-20.

26. Direkze NC, Hodivala-Dilke K, Jeffery R, Hunt T, Poulsom R, Oukrif D, et al. Bone marrow contribution to tumor-associated myofibroblasts and fibroblasts. Cancer Res 2004;64:8492-5.

27. Apte MV, Pirola RC, Wilson JS. Pancreatic stellate cells: a starring role in normal and diseased pancreas. Front Physiol 2012;3:344.

28. von Ahrens D, Bhagat TD, Nagrath D, Maitra A, Verma A. The role of stromal cancer-associated fibroblasts in pancreatic cancer. J Hematol Oncol 2017;10:76.

29. Bulle A, Lim K-H. Beyond just a tight fortress: contribution of stroma to epithelial-mesenchymal transition in pancreatic cancer. Signal Transduct Target Ther 2020;5:249.

30. Bachem MG, Schünemann M, Ramadani M, Siech M, Beger $\mathrm{H}$, Buck A, et al. Pancreatic carcinoma cells induce fibrosis by stimulating proliferation and matrix synthesis of stellate cells. Gastroenterology 2005;128:907-21.

31. Xu Z, Vonlaufen A, Phillips PA, Fiala-Beer E, Zhang X, Yang $\mathrm{L}$, et al. Role of pancreatic stellate cells in pancreatic cancer metastasis. Am J Pathol 2010;177:2585-96.

32. Manoukian P, Bijlsma M, van Laarhoven H. The cellular origins of cancer-associated fibroblasts and their opposing contributions to pancreatic cancer growth. Front Cell Dev Biol 2021;9:743907.

33. Kikuta K, Masamune A, Watanabe T, Ariga H, Itoh H, Hamada $\mathrm{S}$, et al. Pancreatic stellate cells promote epithelial-mesenchymal transition in pancreatic cancer cells. Biochem Biophys Res Commun 2010;403:380-4.

34. Hyun K-A, Koo G-B, Han H, Sohn J, Choi W, Kim S-I, et al. Epithelial-to-mesenchymal transition leads to loss of EpCAM and different physical properties in circulating tumor cells from metastatic breast cancer. Oncotarget 2016;7:24677-87.

35. Maier HJ, Wirth T, Beug H. Epithelial-mesenchymal transition in pancreatic carcinoma. Cancers 2010;2:2058-83.

36. Palamaris K, Felekouras E, Sakellariou S. Epithelial to mesenchymal transition: key regulator of pancreatic ductal adenocarcinoma progression and chemoresistance. Cancers 2021;13:5532.

37. Sada M, Ohuchida K, Horioka K, Okumura T, Moriyama T, Miyasaka Y, et al. Hypoxic stellate cells of pancreatic cancer 
stroma regulate extracellular matrix fiber organization and cancer cell motility. Cancer Lett 2016;372:210-8.

38. Nemolato S, Restivo A, Cabras T, Coni P, Zorcolo L, Orrù G, et al. Thymosin $\beta 4$ in colorectal cancer is localized predominantly at the invasion front in tumor cells undergoing epithelial mesenchymal transition. Cancer Biol Ther 2012;13:191-7.

39. Haeberle L, Cacciato Insilla A, Kapp A-C, Steiger K, Schlitter AM, Konukiewitz B, et al. Stroma composition and proliferative activity are related to therapy response in neoadjuvant treated pancreatic ductal adenocarcinoma. Histol Histopathol 2021;36:733-42.

40. Dardare J, Witz A, Merlin J-L, Bochnakian A, Toussaint P, Gilson $\mathrm{P}$, et al. Epithelial to mesenchymal transition in patients with pancreatic ductal adenocarcinoma: State-of-the-art and therapeutic opportunities. Pharmaceuticals (Basel) 2021; $14: 740$.

41. Stark AP, Sacks GD, Rochefort MM, Donahue TR, Reber HA, Tomlinson JS, et al. Long-term survival in patients with pancreatic ductal adenocarcinoma. Surgery 2016;159:1520-7.

42. He C, Zhang Y, Cai Z, Lin X, Li S. Overall survival, and cancer-specific survival in patients with surgically resected pancreatic head adenocarcinoma: A competing risk nomogram analysis. J Cancer 2018;9:3156-67.

43. Sinn M, Denkert C, Striefler JK, Pelzer U, Stieler JM, Bahra $\mathrm{M}$, et al. $\alpha$-smooth muscle actin expression and desmoplastic stromal reaction in pancreatic cancer: results from the CONKO-001 study. Br J Cancer 2014;111:1917-23.

44. Haeberle L, Steiger K, Schlitter AM, Safi SA, Knoefel WT, Erkan M, et al. Stromal heterogeneity in pancreatic cancer and chronic pancreatitis. Pancreatology 2018;18:1-14.

45. Fong D, Steurer M, Obrist P, Barbieri V, Margreiter R, Amberger A, et al. Ep-CAM expression in pancreatic and ampullary carcinomas: frequency and prognostic relevance. J Clin Pathol 2008;61:31-5.

46. Karamitopoulou E, Zlobec I, Born D, Kondi-Pafiti A, Lykoudis P, Mellou A, et al. Tumour budding is a strong and independent prognostic factor in pancreatic cancer. Eur J Cancer 2013;49:1032-9.

47. Matros E, Bailey G, Clancy T, Zinner M, Ashley S, Whang E, et al. Cytokeratin 20 expression identifies a subtype of pancreatic adenocarcinoma with decreased overall survival. Cancer 2006;106:693-702.

48. Myoteri D, Dellaportas D, Lykoudis PM, Apostolopoulos A, Marinis A, Zizi-Sermpetzoglou A. Prognostic evaluation of vimentin expression in correlation with Ki67 and CD44 in surgically resected pancreatic ductal adenocarcinoma. Gastroenterol Res Pract 2017;2017;1-7.

49. Muckenhuber A, Berger AK, Schlitter AM, Steiger K, Konukiewitz B, Trumpp A, et al. Pancreatic ductal adenocarcinoma subtyping using the biomarkers hepatocyte nuclear factor-1A and cytokeratin- 81 correlates with outcome and treatment response. Clin Cancer Res 2018; 24:351-9.

50. Bray F, Ferlay J, Soerjomataram I, Siegel RL, Torre LA, Jemal A. Global cancer statistics 2018: GLOBOCAN estimates of incidence and mortality worldwide for 36 cancers in 185 countries. CA Cancer J Clin 2018;68:394-424.

51. Bartlett JE, Kotrlik JW, Higgins CC. Organizational research: Determining appropriate sample size in survey research. Inf
Technol Learn Perform J 2001;19:43-50.

52. Pacheco-Velázquez SC, Gallardo-Pérez JC, Díaz D, AdánLadrón de Guevara A, Robledo-Cadena DX, Saavedra E, et al. Heart myxoma develops oncogenic and metastatic phenotype. J Cancer Res Clin Oncol 2019;145:1283-95.

53. Chan KS, Ho BCS, Shelat VG. A pilot study of estrogen receptor (ER) expression in pancreatic ductal adenocarcinoma (PDAC). Transl Gastroenterol Hepatol 2021;6:9.

54. Hassan S, Ferrario C, Mamo A, Basik M. Tissue microarrays: emerging standard for biomarker validation. Curr Opin Biotechnol 2008;19:19-25.

55. Santosh N, McNamara KK, Beck FM, Kalmar JR. Expression of cornulin in oral premalignant lesions. Oral Surg Oral Med Oral Pathol Oral Radiol 2019;127:526-34.

56. Marinaccio C, Ribatti D. A simple method of image analysis to estimate CAM vascularization by APERIO ImageScope software. Int J Dev Biol 2015;59:217-9.

57. Diaz D, Vazquez-Polanco AM, Argueta-Donohue J, Stephens CR, Jimenez-Trejo F, Ceballos-Liceaga SE, et al. Incidence of Intestinal infectious diseases due to protozoa and bacteria in Mexico: Analysis of national surveillance records from 2003 to 2012. BioMed Res Int 2018;2018:2893012.

58. Whitaker-Menezes D, Martinez-Outschoorn UE, Flomenberg N, Birbe RC, Witkiewicz AK, Howell A, et al. Hyperactivation of oxidative mitochondrial metabolism in epithelial cancer cells in situ: visualizing the therapeutic effects of metformin in tumor tissue. Cell Cycle 2011;10:4047-64.

59. Maehira H, Miyake T, Iida H, Tokuda A, Mori H, Yasukawa D, et al. Vimentin expression in tumor microenvironment predicts survival in pancreatic ductal adenocarcinoma: Heterogeneity in fibroblast population. Ann Surg Oncol 2019;26:4791-804.

60. Wang M, Estrella JS, Katz MH, Kim M, Rashid A, Lee JE, et al. Expression of epithelial-mesenchymal transition markers in treated pancreatic ductal adenocarcinoma. Pancreas 2019;48:1367-72.

61. Javle MM, Gibbs JF, Iwata KK, Pak Y, Rutledge P, Yu J, et al. Epithelial-mesenchymal transition (EMT) and activated extracellular signal-regulated kinase (p-Erk) in surgically resected pancreatic cancer. Ann Surg Oncol 2007;14:3527-33.

62. Dhayat SA, Traeger MM, Rehkaemper J, Stroese AJ, Steinestel $\mathrm{K}$, Wardelmann E, et al. Clinical impact of epithelial-to-mesenchymal transition regulating microRNAs in pancreatic ductal adenocarcinoma. Cancers 2018;10:1-20.

63. Kohler I, Bronsert P, Timme S, Werner M, Brabletz T, Hopt UT, et al. Detailed analysis of epithelial-mesenchymal transition and tumor budding identifies predictors of long-term survival in pancreatic ductal adenocarcinoma. J Gastroenterol Hepatol 2015;30:S78-84.

64. Chouat E, Zehani A, Chelly I, Njima M, Maghrebi H, Bani $\mathrm{MA}$, et al. Tumor budding is a prognostic factor linked to epithelial mesenchymal transition in pancreatic ductal adenocarcinoma. Study report and literature review. Pancreatology 2018; $18: 79-84$

65. Hu D, Ansari D, Zhou Q, Sasor A, Said Hilmersson K, Andersson R. Stromal fibronectin expression in patients with resected pancreatic ductal adenocarcinoma. World J Surg Oncol 2019;17:29.

Received for publication: 9 November 2021. Accepted for publication: 9 February 2022.

This work is licensed under a Creative Commons Attribution-NonCommercial 4.0 International License (CC BY-NC 4.0).

(C) Copyright: the Author(s), 2022

Licensee PAGEPress, Italy

European Journal of Histochemistry 2022; 66:3360

doi:10.4081/ejh.2022.3360

[European Journal of Histochemistry 2022; 66:3360] 